\title{
Molecular Design of Anticancer Drug from Marine Fungi Derivatives
}

\author{
Duc Tuan Cao, ${ }^{a}$ Thi Mai Huong Doan, ${ }^{b}$ Van Cuong Pham, ${ }^{b}$ Thi Hong Lien Hoang, ${ }^{c}$ Jung-Woo Chae, ${ }^{d}$ Hwi- \\ yeol Yun, ${ }^{d}$ Min-Kyun Na, ${ }^{d}$ Young-Ho Kim, ${ }^{d}$ Minh Quan Pham, ${ }^{e, f^{*}}$ and Van Hung Nguyen ${ }^{a^{*}}$ \\ a. Hai Phong University of Medicine and Pharmacy, Haiphong, Vietnam; Email: nvhung@hpmu.edu.vn \\ b. Institute of Marine Biochemistry, Vietnam Academy of Science and Technology, Hanoi, Vietnam \\ c. Buon Ma Thuot University, Buon Ma Thuot, Dak Lak, Vietnam \\ d. College of Pharmacy, Chungnam National University, Daejeon, Republic of Korea \\ e. Institute of Natural Products Chemistry, Vietnam Academy of Science and Technology, Hanoi, \\ Vietnam; Email: pham-minh.quan@inpc.vast.vn \\ f.Graduate University of Science and Technology, Vietnam Academy of Science and Technology, \\ Hanoi, Vietnam
}

\begin{abstract}
Heat shock protein 90 (HSP90) is known as one of the most potential target in cancer therapy. In this context, we have demonstrated that marine fungi derivatives can play as possible inhibitors for preventing the biological activity of HSP9O using a combination of molecular docking and fast pulling of ligand (FPL) simulations. In particular, the computational approaches were validated since compared with the respective experiments. Based on a benchmark on available inhibitors of HsP90, GOLD docking package using ChemPLP scoring function was found to be dominated over both Autodock Vina and Autodock4 in preliminary estimation the ligand binding affinity and binding pose with the Pearson correlation, $R=-0.62$. Moreover, FPL calculations were also indicated to be a suitable approach to refine docking simulations with a correlation coefficient with the respective experimental data of $R=-0.81$. Therefore, the binding affinity of marine fungi derivatives to $\mathrm{Hsp} 90$ was evaluated. Docking and FPL calculations suggested that five compounds including $\mathbf{2 3}, \mathbf{4 0}, \mathbf{4 6}, \mathbf{4 8}$, and 52 are as highly potential inhibitors for HSP90. The obtained results probably enhance the cancer therapy.
\end{abstract}

\section{Introduction}

The heat shock protein family including Hsp27, Hsp70, Hsp90, etc are crucial elements in cell homeostasis. ${ }^{1-3}$ In particular, Hsp90 is a dedicated chaperone, occupies ca. $2 \%$ of total protein content, reacting with ca. 200 various proteins. These proteins are a vital part of constitutional cell signaling and accommodative reply to stress. The Hsp90 is thus characterized that it is probably associated with six stamps of cancer. ${ }^{3,4}$ Consequently, the enzyme is associated with the cancer cell survival. ${ }^{4}$ Therefore, $\mathrm{Hsp} 90$ is distinguished as a target for cancer therapy. ${ }^{5}$ Several studies were thus executed to discover the highly potential inhibitors for preventing the biological activity of Hsp90. 6-14

Since the dawn of the human kind, nature has been considered as the main source providing folk remedies and therapeutics agents for the treatment of a wide spectrum of diseases. It is estimated that among all the medicines being provided on the international market, more than $60 \%$ of them are derived from or inspired by natural products, mainly originated from terrestrial plants. ${ }^{15}$ On the other hand, in the last 50 years, with the advances in new technologies and engineering in marine science, scientists are increasing shift their focus toward marine organisms. Numerous publication in this field has proved that marine organisms provide many natural compounds with biological activities ranging from antiviral to anticancer for the pharmaceutical industries. ${ }^{16,17}$ In addition, the uncommon and unique of marine chemical structures could be scaffold for developing new drugs with greater efficacy and specificity for the therapeutics. ${ }^{18}$ During the last decades, a reduced number of novel compounds isolated from macro-organisms 
were observed and microorganisms such as marine fungi and bacteria represent promising sources with several hits have been found from the drug discovery. ${ }^{19,20}$ In this light, marine fungi are important not only from the perspective of new drugs but also having the advantage of sustainable production of large quantities of compounds with reasonable costs and large scale cultivation. Based on information above, in this study, a set of compounds originated from marine fungi will be investigated to look for hits with Hsp90 potential inhibition activity.

Recently, the computer-aided drug design (CADD) is widely used to decrease both cost and time for developing a novel therapy. ${ }^{21,22}$ In CADD, the thermodynamics metric $\Delta G$, corresponds to the binding free energy between protein and ligand, is able to compute over atomistic simulations. This metric is associated with the experimental data via formula $\Delta G_{\text {bind }}=R T \ln \left(k_{\mathrm{i}}\right)$, where $R$ is gas constant, $T$ is absolute temperature, $k_{\mathrm{i}}$ is inhibition constant. Popularly, the experimental data $I C_{50}$ is probably assumed to be equivalent to $k_{\mathrm{i}}$ in order to characterize the experimental binding free energy $\Delta G_{\mathrm{EXP}} .^{23-25}$ Because the metric discloses the inhibitor efficiency, ${ }^{21}$ accurate and speedy determination of the $\Delta G$ is very important in discovery of potential inhibitors for a protein target. ${ }^{26-31}$ In this work, we have first benchmarked the performance of the free energy approaches including molecular docking and FPL calculations. The obtained results in estimation the $\Delta G$ of marine fungi derivatives is thus more reliable. Compound 23, 40, 46, 48, and 52 were suggest that they are highly possible inhibitors for preventing the biological activity of HSP90. The observation can boost the cancer treatment.

\section{Materials and Methods}

\section{Structure of Ligands and HSP90}

The three-dimensional structures of HSP90 was downloaded from the Protein Data Bank (PDB) with the identify (ID) of 3K99. ${ }^{10}$ The resolution of HSP9O is relatively high with an among of 1.79 $\AA$. The available inhibitors for HSP90 were also obtained via PDB database with ID: $2 \mathrm{QF6},{ }^{9}$ 2QG0, ${ }^{9}$ 2QG2, ${ }^{9} 3 \mathrm{~K} 97,{ }^{10} 3 \mathrm{~K} 99,{ }^{10} 3 \mathrm{R} 4 \mathrm{M},{ }^{11} 3 \mathrm{R} 4 \mathrm{~N},{ }^{11} 3 \mathrm{R} 4 \mathrm{O},{ }^{11} 3 \mathrm{RLR},{ }^{12} 4 \mathrm{NH} 7,{ }^{13} 4 \mathrm{NH} 8,{ }^{13} 4005,{ }^{14} 4007,{ }^{14} 4009,{ }^{14}$ and $400 B{ }^{14}$ The 3D structure of 63 ligands including compounds 1-52 were obtained via our previous investigations on marine fungi samples (Aspergillus sp. and Penicillium sp.) ) $^{32-34}$ and compounds 53-63 in unpublished work by Cao et al. The structure of compounds are shown in the electronic supplementary (ESI) file.

\section{Molecular Docking Simulations}

The molecular docking simulations were employed to initial evaluation both binding affinity and pose of ligands to HSP90. Here we employed Autodock Vina (Vina) $1.1,35$ Autodock4.2 (AD4), ${ }^{36}$ and GOLD packages ${ }^{37}$ to finish the task force. The parameters of the docking simulations via AD4 and Vina were referred to the previous studies. ${ }^{24,}{ }^{33}$ Whereas GOLD parameters were selected as default options. The best docking structure is selected as the docking configuration forming the highest binding affinity.

Vina docking. ${ }^{35}$ The docking simulation was performed using parameters referred to the previous studies. $33,38,39$ In particular, the exhaustiveness is chosen as 8 and the maximum of different energy between docking configurations is selected as $7 \mathrm{kcal} \mathrm{mol}^{-1}$. The grid size of the Vina docking is selected as $20 \times 20 \times 20 \AA$ with the center of grid is chosen as the center of mass of the experimental ligand (cf. Error! Reference source not found.).

AD4 docking. ${ }^{36}$ AD4 was executed with docking parameters referred to the previous studies. ${ }^{33}$ Particularly, grid size was selected as $60 \times 60 \times 60$ with the spacing of $0.333 \AA$ (cf. Error! Reference source not found.). The Autogrid4 was the executed to generate the grid map. Ligands were docked to the HSP90 with the genetic algorithm (GA)/ Lamarckian GA (LA)/Monte Carlo Simulated Annealing (SA) number of evaluations was 250.000, equivalent to short option. Moreover, the GA run is of 10, the population size is of 150, and the number of generations is of 27.000.

GOLD docking. ${ }^{37}$ The molecular docking simulation was executed using GOLD docking package referring to the previous work. ${ }^{40}$ The grid center was selected as well as the AD4/Vina parameters. 
All atoms within the range of $12 \AA$ would be considered during docking simulations. The GOLD docking package was performed using two scoring functions as well as ChemScore and ChemPLP.

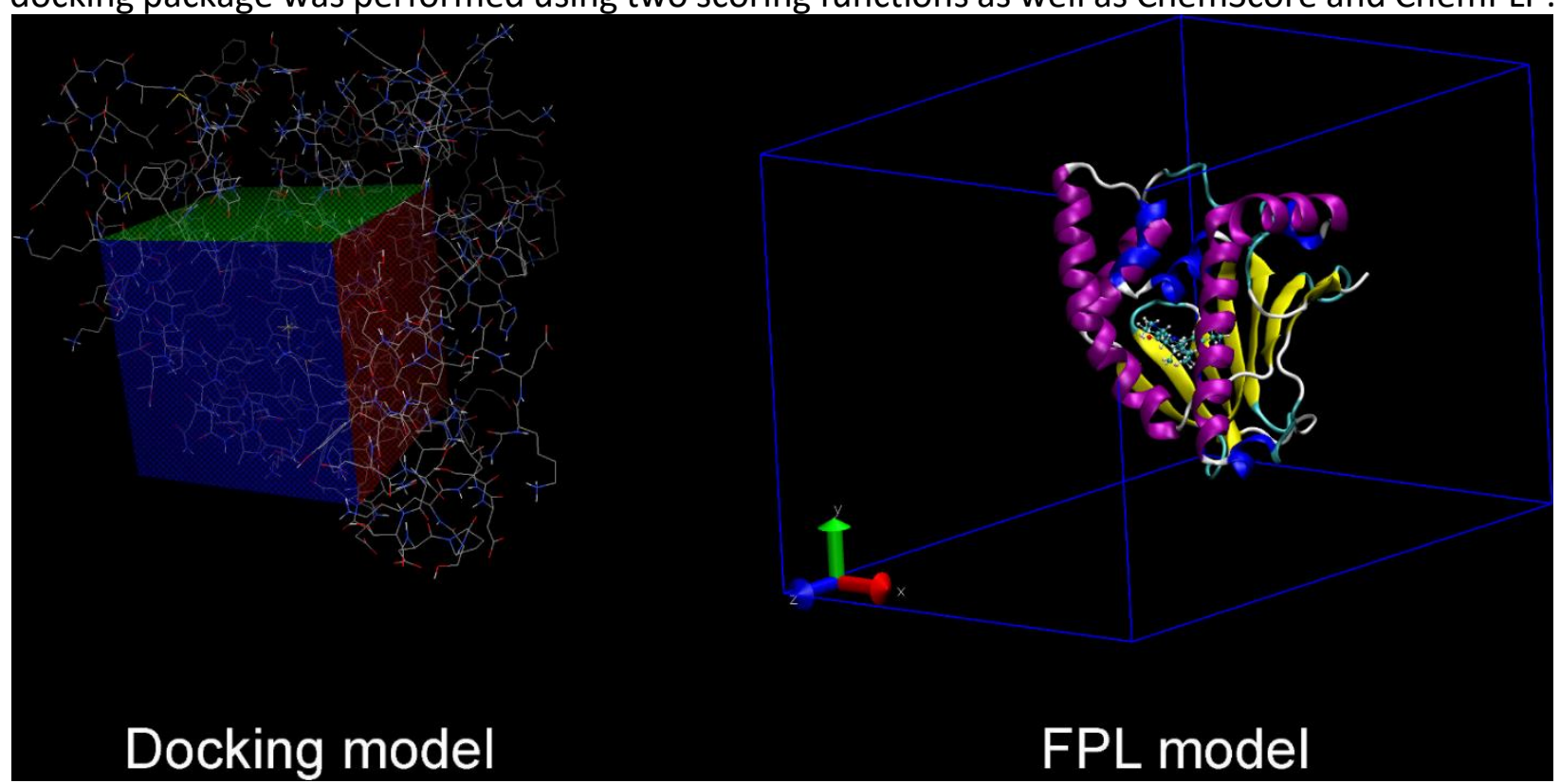

Figure 1. Computational modelling of molecular docking and FPL simulations.

\section{Fast Pulling of Ligand (FPL) Simulations}

GROMACS version $5.1 .5^{41}$ was employed to mimic the unbinding process of a ligand, which was pulled via an external force, mobilizing out of the binding cavity of the HSP90. The HSP90 and counteracted ions were presented by using the Amber99SB-ILDN force field (FF). ${ }^{42}$ The TIP3P was engaged for treating water molecules. ${ }^{43}$ The general Amber force field (GAFF) ${ }^{44}$ was utilized to parameterize the ligand via AmberTools18 with the charged and geometric parameters obtaining via QM calculations using B3LYP functional and 6-31G(d,p) basis set. ${ }^{45}$ In particular, the atomic charge was evaluated using the restrained electrostatic potential (RESP) method. ${ }^{46}$ The ACPYPE ${ }^{47}$ protocol was then utilized to convert the ligand topology to GROMACS formats. The combined FF was selected since they are high coincidence for free energy assessment. ${ }^{48,49}$

The HSP9O + ligand complex was initially inserted into a rectangular periodic boundary condition box with a size of $5.8 \times 5.8 \times 8.5 \mathrm{~nm}$, which is large enough for unbinding ligand out of enzymic binding cavity (cf. Error! Reference source not found.). The soluble complex thus comprises of ca. 27500 atoms involving the HSP90, ligands, water molecules, and ca. $7 \mathrm{Na}^{+}$ions. The steepest descent approach was initially utilized for energy minimizing the system. Then, NVT and NPT imitations were then followed to relax the system with a length of $0.1 \mathrm{~ns}$ each simulation. It should be noted that the $C_{\alpha}$ atoms of HSP9O were positionally restrained using a weak harmonic potential. Finally, the inhibitor was stressed to mobilize out of the HSP binding cavity by utilizing an external harmonic force with a pulling speed of $k=0.005 \mathrm{~nm} \mathrm{ps}^{-1}$ and a spring constant of $v=600 \mathrm{~kJ} \mathrm{~mol}^{-1} \mathrm{~nm}^{-2}$. The metrics $k$ and $v$ were chosen as referred to the previous works. ${ }^{27}$ During FPL calculations, the displacement of ligand and the value of pulling force, $F$, were recorded every $0.1 \mathrm{ps}$ that would be used to estimate the ligand binding affinity. ${ }^{27}$ The FPL calculations were carried out 8 times each system.

Analyzed Tools

The ligand protonation state was predicted by using the Chemicalize tools (www.chemicalize.com), a website application of the ChemAxon. The binding pose of ligand to HSP90 was analyzed using the free version of Maestro 2020, ${ }^{50}$ and PyMOL 2.4.0. ${ }^{51}$

\section{Results and Discussion}




\section{Molecular Docking Simulation}

In CADD, the binding pose and binding affinity of ligands to enzymic target are generally obtained via molecular docking simulation, a fast and low computing resources technique. In this work, we have tried Vina, ${ }^{35}$ an open-source docking protocol, which is widely used for this purpose to complete this task force. Unfortunately, Vina calculations adopted uncorrelated results in compared with the respective experimental data since the correlation coefficient $R_{\text {Vina }}=0.40$ (Error! Reference source not found.). AD4 was thus employed to perform the docking simulation since they have a different scoring function to Vina. ${ }^{35,36}$ Because Vina uses a full empirical scoring function, while AD4 uses a semi-physical/empirical based scoring function. ${ }^{35}$, ${ }^{36}$ We thus expected that AD4 probably is suitable for completing the task force that the obtained docking result would be appropriate. Unfortunately, the obtained correlation coefficient is poor with a value of $R_{\mathrm{AD} 4}^{\mathrm{GA}}=0.33$ (Error! Reference source not found.). It is in good consistent with the previous work. ${ }^{33}$ Although we have changed search algorithms from GA to Lamarckian GA (LA) and Monte Carlo Simulated Annealing (SA), the poor correlated results were observed with a value of $R_{\mathrm{AD} 4}^{\mathrm{LA}}=0.35$ and $R_{\mathrm{AD} 4}^{\mathrm{SA}}=0.25$ (Error! Reference source not found.).

The GOLD docking package, ${ }^{37}$ a commercial docking progam, was then utilized to evaluate the binding affinity and pose of ligands to HSP9O enzyme. Fortunately, the docking energy provided by GOLD docking package forms appropriate correlations with the respective experiment with a value of $R_{\mathrm{GOLD}}^{\mathrm{ChemScore}}=0.51$ and $R_{\mathrm{GOLD}}^{\mathrm{ChemPLP}}=-0.60$ (cf. Error! Reference source not found. and Figure 1). Absolutely, ChemPLP scoring function is much better than ChemScore one and the ligand having higher ChemPLP score will form a higher binding affinity to Hsp90. Therefore, GOLD docking package with ChemPLP scoring function was executed to predict the binding affinity of marine derivatives toward Hsp90.

Table 1. The obtained values of the docking simulations.

\begin{tabular}{|c|c|c|c|c|c|c|c|c|}
\hline $\mathbf{N}^{0}$ & PDB ID & $\Delta \boldsymbol{G}_{\text {Vina }}$ & $\Delta G_{\mathrm{AD} 4}^{\mathrm{GA}}$ & $\Delta G_{\mathrm{AD} 4}^{\mathrm{LA}}$ & $\Delta G_{\mathrm{AD} 4}^{\mathrm{SA}}$ & $\Delta G_{\mathrm{GOLD}}^{\text {ChemScore }}$ & ChemPLP & $\Delta \boldsymbol{G}_{\mathrm{EXP}}{ }^{\mathrm{a}}$ \\
\hline 1 & $2 \mathrm{QF6}$ & -10.2 & -8.8 & -8.8 & -8.8 & -8.8 & 74.7 & -8.91 \\
\hline 2 & $2 Q G 0$ & -9.6 & -8.7 & -9.2 & -10.4 & -7.0 & 66.4 & -7.85 \\
\hline 3 & $2 \mathrm{QG} 2$ & -10.2 & -9.9 & -9.4 & -10.0 & -7.9 & 75.2 & -7.41 \\
\hline 4 & 3К97 & -8.3 & -7.7 & -7.9 & -8.1 & -7.3 & 68.3 & -10.98 \\
\hline 5 & 3К99 & -9.2 & -8.2 & -8.2 & -8.2 & -7.9 & 61.4 & -9.91 \\
\hline 6 & $3 R 4 M$ & -7.8 & -6.6 & -6.6 & -7.5 & -5.1 & 49.7 & -8.13 \\
\hline 7 & $3 R 4 N$ & -8.3 & -7.1 & -7.4 & -7.6 & -5.4 & 61.2 & -9.45 \\
\hline 8 & $3 R 40$ & -10.1 & -8.8 & -8.7 & -9.2 & -6.0 & 73.2 & -11.19 \\
\hline 9 & $3 R L R$ & -9.6 & -8.2 & -8.2 & -8.2 & -6.4 & 70.5 & -10.33 \\
\hline 10 & $4 \mathrm{NH} 7$ & -11.3 & -10.0 & -9.9 & -10.6 & -11.8 & 98.9 & -11.53 \\
\hline 11 & $4 \mathrm{NH} 8$ & -10.7 & -9.7 & -9.8 & -10.0 & -11.8 & 98.3 & -11.70 \\
\hline 12 & 4005 & -12.5 & -11.5 & -11.4 & -11.5 & -11.4 & 96.4 & -10.20 \\
\hline 13 & 4007 & -12.5 & -11.9 & -11.9 & -12.0 & -11.5 & 95.5 & -10.13 \\
\hline 14 & 4009 & -11.6 & -10.7 & -10.7 & -10.8 & -11.0 & 93.8 & -10.57 \\
\hline 15 & $400 B$ & -12.3 & -11.5 & -11.7 & -11.7 & -11.1 & 94.2 & -11.39 \\
\hline
\end{tabular}




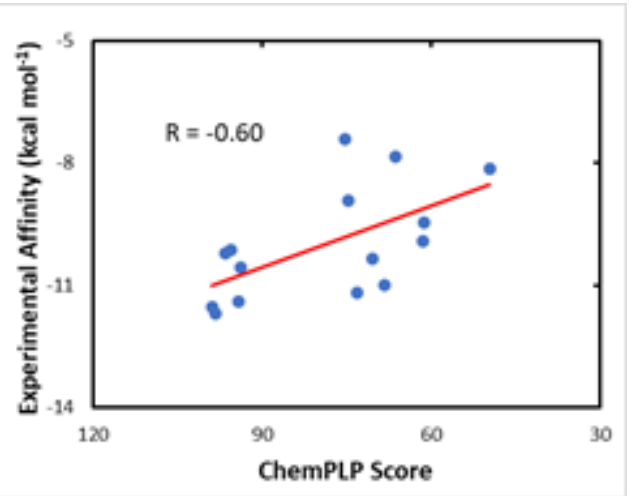

Figure 1. Association between the ChemPLP score and the experimental binding free energy FPL Simulations

$$
\Delta G_{\mathrm{EXP}}
$$

Although the docking simulation adopted well-appropriate results compared with the respected experimental data, ${ }^{52-54}$ the docking results are required to refine via a more accurate approach. ${ }^{39,55,56}$ Because the molecular docking approach normally uses many constraints to enhance the computing speed. The popular constraints of molecular docking simulations are known as lacking receptor dynamics, limiting number trial position of ligands, using implicit water model, etc. Normally, molecular dynamics and/or steered-molecular dynamics simulations were thus utilized to refine the docking outcomes. ${ }^{28,57,58}$ In this context, FPL simulations were employed to validate the docking results since is a very efficient free energy approach, which normally form appropriate results compared to the respective experiments with low computing resources. ${ }^{59} \mathrm{FPL}$ were thus successfully applied on various targets before. ${ }^{27,} 39,59$

In this context, we first benchmarked the performance of FPL scheme over 15 available inhibitors-Hsp90 complex systems. The obtained results were shown in the Error! Reference source not found. and Table S2 of the ESI file. In particular, the mean of pulling work $(W)$ diffuses in the range from 58.3 to $208.5 \mathrm{kcal} \mathrm{mol}^{-1}$, whereas the mean of rupture force $\left(F_{\text {Max }}\right)$ adopts in the range from 580.7 to $1566.1 \mathrm{pN}$. The graph of $W$ and $F_{\mathrm{Max}}$ in time revolution was described in the electronic supplementary (ESI) file. The computed metrics are in good consistent with the respective experimental data, ${ }^{9-14}$ because Pearson correlation coefficient is of $R_{\mathrm{W}}=-0.78$ and $R_{\mathrm{F}}=-0.81$ (Error! Reference source not found. and Figure 2). Although the difference between $R_{\mathrm{W}}$ and $R_{\mathrm{F}}$ is quite small, the rupture force was selected to rank the ligand-binding affinity to Hsp90. Therefore, using linear regression, the predicted binding free energy $\Delta G_{\mathrm{FPL}}^{\mathrm{Pre}}$ can be calculated via the rupture force as follow :

$$
\Delta G_{\mathrm{FPL}}^{\mathrm{Pre}}=-0.3345 * \frac{F_{\mathrm{Max}}}{100}-6.523
$$

The metric $\Delta G_{\mathrm{FPL}}^{\mathrm{Pre}}$ was investigated by the RMSE with linear regression, forming $R M S E_{\mathrm{F}}=0.77$ $\mathrm{kcal} \mathrm{mol}^{-1}$, which imply that FPL calculation can categorize ligands having similar binding affinity to Hsp90. Overall, FPL simulation is an efficient approach to predict the ligand-binding free energy of Hsp90.

Table 2. The obtained values of the FPL calculations in comparison with the respective experiments.

\begin{tabular}{ccccc}
\hline $\mathbf{N}^{0}$ & Name & $\boldsymbol{F}_{\text {Max }}{ }^{\mathrm{a}}$ & $\boldsymbol{W}^{\mathbf{b}}$ & $\Delta \mathbf{G}_{\mathbf{E X P}}{ }^{\mathbf{c}}$ \\
\hline 1 & $2 \mathrm{QF6}$ & $580.7 \pm 90.9$ & $67.7 \pm 12.3$ & -8.91 \\
2 & $2 \mathrm{QG0}$ & $764.3 \pm 119.3$ & $76.5 \pm 12.9$ & -7.85 \\
3 & $2 \mathrm{QG2}$ & $676.2 \pm 78.5$ & $71.1 \pm 8.7$ & -7.41 \\
\hline
\end{tabular}




\begin{tabular}{lcccc}
4 & 3 K 97 & $1065.5 \pm 66.3$ & $113.9 \pm 10$ & -10.98 \\
5 & 3 KK99 & $831.4 \pm 52.5$ & $82.7 \pm 4.5$ & -9.91 \\
6 & $3 R 4 M$ & $605.7 \pm 81.7$ & $58.3 \pm 9.4$ & -8.13 \\
7 & $3 R 4 N$ & $651.0 \pm 37.6$ & $69.8 \pm 4.4$ & -9.45 \\
8 & $3 R 40$ & $1140.8 \pm 128.1$ & $126.3 \pm 17.2$ & -11.19 \\
9 & $3 R L R$ & $910.8 \pm 88.1$ & $98.8 \pm 8.6$ & -10.33 \\
10 & $4 \mathrm{NH7}$ & $1357.1 \pm 146.7$ & $167.6 \pm 28$ & -11.53 \\
11 & $4 \mathrm{NH} 8$ & $1566.1 \pm 124.2$ & $208.5 \pm 26.1$ & -11.70 \\
12 & 4005 & $1313.2 \pm 88.2$ & $164.4 \pm 20.3$ & -10.20 \\
13 & 4007 & $1363.3 \pm 131.0$ & $175.7 \pm 25.9$ & -10.13 \\
14 & 4009 & $1348.6 \pm 102.6$ & $163.9 \pm 18.6$ & -10.57 \\
15 & $400 B$ & $1318.9 \pm 112.6$ & $157.5 \pm 20.8$ & -11.39 \\
\hline
\end{tabular}

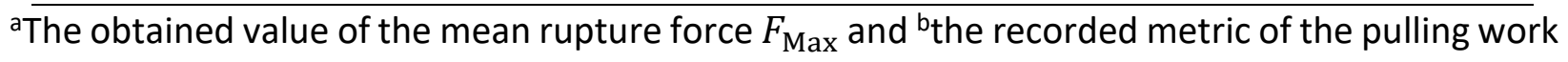
$W$. 'The experimental binding free energy $\Delta G_{\mathrm{EXP}}$ was estimated via the reported $K_{\mathrm{i}}$. The calculated error was the standard error of the average. The unit of force and energy is in $\mathrm{pN}$ and $\mathrm{kcal} \mathrm{mol}^{-1}$, respectively.

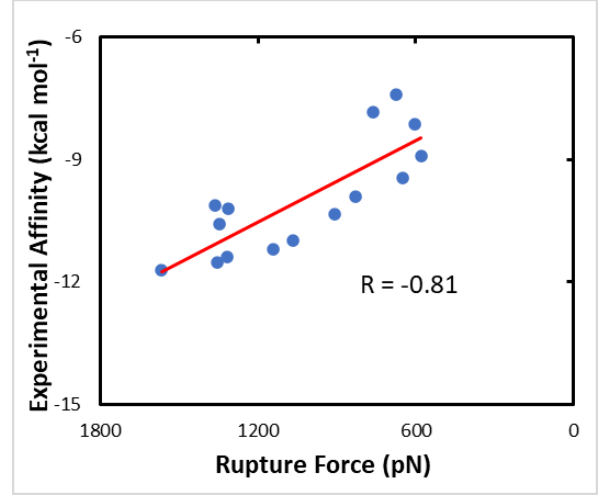

Figure 2. Association between the average of the $F_{\mathrm{Max}}$ and the experimental binding free energy $\Delta G_{\mathrm{EXP}}$.

\section{Marine fungi derivatives inhibit Hsp90}

As mentioned above, marine fungi derivatives are vital compounds that probably plays as promising candidates preventing the biological activity of Hsp90. The GOLD docking package using ChemPLP scoring function was thus utilized to estimate the binding affinity and binding pose of marine fungi derivatives were preliminarily calculated using this application. The obtained results were reported in Table 1 and Table S1 of the ESI file. In particular, the ChemPLP scores of these compounds vary in the range from 31.99 to 77.8. The compounds having ChemPLP scores higher than 70.0 would be proposed as potential inhibitors for preventing Hsp90 (cf. Table 1).

Table 1. The obtained values of the molecular docking and FPL calculations.

\begin{tabular}{ccccccc}
\hline $\mathbf{N}^{0}$ & Name & ChemPLP & $\boldsymbol{F}_{\text {Max }}{ }^{\mathrm{a}}$ & $\boldsymbol{W}^{\mathbf{b}}$ & $\Delta \boldsymbol{G}_{\mathrm{FPL}}^{\text {Pre }^{\mathbf{c}}}$ & $\boldsymbol{k}_{\mathrm{i}}^{\text {Pre }}$ \\
1 & $\mathbf{4 8}$ & 86.3 & $627 \pm 127.1$ & $70.6 \pm 9.2$ & -8.62 & 0.29 \\
2 & $\mathbf{2 5}$ & 77.8 & $555.3 \pm 60.5$ & $61.7 \pm 8.2$ & -8.38 & 1.25
\end{tabular}




$\begin{array}{llllcll}3 & \mathbf{4 0} & 76.9 & 711.7 \pm 77.9 & 75.7 \pm 13 & -8.90 & 0.54 \\ 4 & \mathbf{2 3} & 72.6 & 827.6 \pm 195 & 95.9 \pm 22.3 & -9.29 & 0.67 \\ 5 & \mathbf{4 6} & 71.7 & 669.9 \pm 97.2 & 78.1 \pm 9.8 & -8.76 & 0.85 \\ 6 & \mathbf{5 2} & 70.6 & 753.3 \pm 110 & 77.5 \pm 10.5 & -9.04 & 0.43\end{array}$

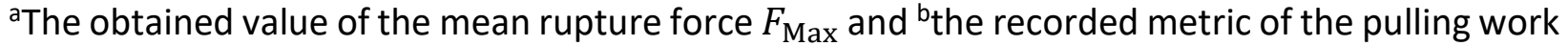
$W .{ }^{c}$ The predicted binding free energy $\Delta G_{\mathrm{FPL}}^{\mathrm{Pre}}$ was estimated via formula (1). The calculated error was the standard error of the average. The unit of force, energy, and inhibition constant is in $\mathrm{pN}$, $\mathrm{kcal} \mathrm{mol}^{-1}$ and $\mu \mathrm{M}$, respectively.

The binding poses between six top-lead docking compounds and Hsp90 enzyme were analyzed using PyMOL 2.4.0 and Maestro 2020 package (cf. Figure 4). Interestingly, there is only ligand 23 forming one hydrogen bond to Hsp90, whereas the other compounds just adopt hydrophobic contacts only. The observation implies that the hydrophobic interaction dominates over electrostatic interaction in the binding process of ligands to Hsp90. It is consistent with the correlation between experimental binding free energy with the metric ClogP, $R=-0.64$, which meaning that more hydrophobic inhibitors cruel stronger binding ligands.

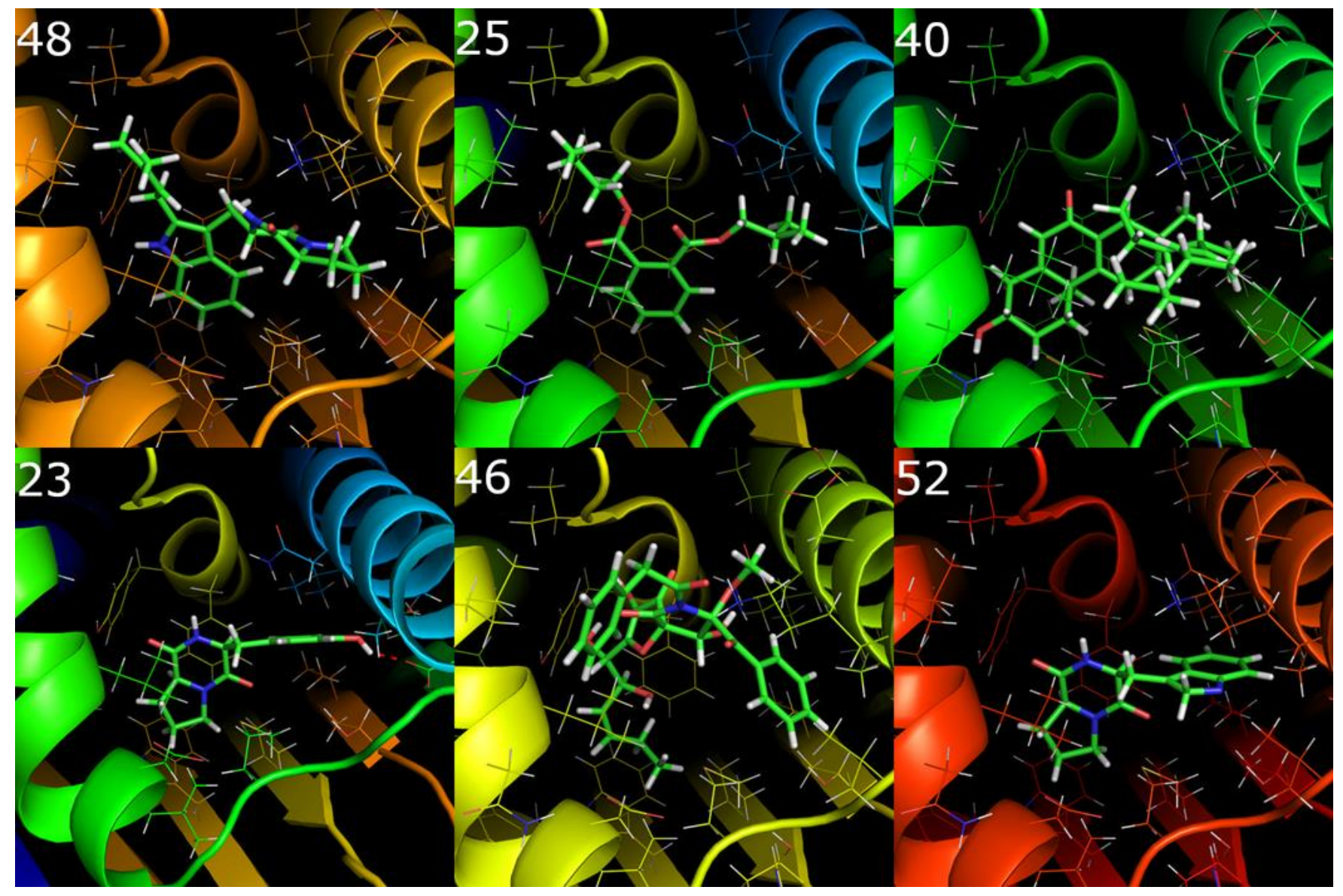

Figure 4. Binding poses of top-lead marine fungi compounds to Hsp90, which was estimated using the GOLD docking program with ChemPLP scoring function.

FPL calculations were then applied to refine the docking results of 63 marine fungi derivatives to Hsp90. The obtained results were shown in Table 1 and Table S3 of the ESI file. The average of $F_{\text {Max }}$ falls in the range from 555.3 to $827.6 \mathrm{pN}$, whereas the mean $W$ falls in the range from 57 to 
$94 \mathrm{kcal} \mathrm{mol}^{-1}$. Using linear regression via formula (1), the predicted binding free energy of six toplead marine compounds were obtained as mentioned in Table 1 . The predicted metrics $\Delta G_{\mathrm{FPL}}^{\mathrm{Pre}}$ diffuse in the range from -8.38 to $-9.23 \mathrm{kcal} \mathrm{mol}^{-1}$, which is equivalent to the predicted inhibition constant $k_{\mathrm{i}}^{\text {Pre }}$ arranging from 0.29 to $1.25 \mu \mathrm{M}$. Interestingly, five ligands adopts a sub-micromolar inhibition constant implying that they probably a promising inhibitors for blocking the biological activity of Hsp90. Additional evaluation using in vitro and/or in vivo studies should be executed to validate the obtained results.

\section{CPU Time Consumption}

Each Hsp90 + inhibitor system was mimicked over 8 independent FPL calculations as well as the performed calculations of the different targets. ${ }^{39,56}$ In particular, different FPL calculations have the same starting structure and one FPL simulation involves $0.1 \mathrm{~ns}$ of NVT, $0.1 \mathrm{~ns}$ of NPT, and $0.5 \mathrm{~ns}$ of SMD simulations. $5.6 \mathrm{~ns}$ of MD simulations was thus carried out to predict the binding free energy of a ligand to Hsp90 target. It should be denoted that a dual Xeon E5-2683 V3 server is able to perform ca. 90 ns of MD simulation each day for Hsp90 + inhibitor complex. Therefore, FPL approach can be used to estimate the binding free energy of a ligand to Hsp90 within 1.5 hours. It is quite low computing resource requirements allowing us to be able to accurately and rapidly evaluate the binding free energy of several ligands to Hsp90.

\section{Conclusions}

In this context, we have established that a amalgamation of GOLD docking and FPL simulations can accurately and rapidly calculate the binding free energy of a ligand to Hsp90. The ChemPLP scoring function is better than ChemScore one in docking a molecule to $\mathrm{Hsp} 90$ since $R_{\mathrm{GOLD}}^{\text {ChemScore }}=$ 0.51 is significantly smaller than $R_{\mathrm{GOLD}}^{\mathrm{ChemPLP}}=-0.60$. Besides that, although, we also tried AD4 and Vina docking to complete the task force, the poor correlation to the respective experiments was observed. Moreover, FPL calculation is an efficient protocol to refine the docking result since forming a good correlation coefficient $R_{\mathrm{F}}=-0.81$ and $R M S E_{\mathrm{F}}=0.77 \mathrm{kcal} \mathrm{mol}^{-1}$ with a low computing resource requirement. Furthermore, based on the efficient of the approaches, a short list comprising five compounds from marine fungi derivative including $\mathbf{2 3}, \mathbf{4 0}, \mathbf{4 6}, \mathbf{4 8}$, and 52 were indicated that they are promising inhibitors for preventing the biological activity of Hsp90 because they adopt a sub-micromolar inhibition constant. Further evaluation using in vitro and/or in vivo studies should be executed to validate the obtained results.

\section{Acknowledgements}

This research was funded by Vietnam Ministry of Science and Technology (Grant number: HNQT/SPĐP/11.19) and College of Pharmacy, Chungnam National University, Republic of Korea under the Vietnam bilateral - multilateral international joint research program in Science and Technology to 2020.

\section{Conflicts of interest}

The author(s) declared no potential conflicts of interest with respect to the research, authorship, and/or publication of this article.

\section{References}

1. C. Jolly and R. I. Morimoto, J. Natl. Cancer Inst, 2000, 92, 1564-1572.

2. F. Vallée, C. Carrez, F. Pilorge, A. Dupuy, A. Parent, L. Bertin, F. Thompson, P. Ferrari, F. Fassy, A. Lamberton, A. Thomas, R. Arrebola, S. Guerif, A. Rohaut, V. Certal, J.-M. Ruxer, C. Delorme, A. Jouanen, J. Dumas, C. Grépin, C. Combeau, H. Goulaouic, N. Dereu, V. Mikol, P. Mailliet and H. Minoux, J. Med. Chem, 2011, 54, 7206-7219. 
3. A. J. L. Macario and E. C. de Macario, N Engl J Med, 2005, 353, 1489-1501.

4. D. Hanahan and R. A. Weinberg, Cell, 2000, 100, 57-70.

5. D. Mahalingam, R. Swords, J. S. Carew, S. T. Nawrocki, K. Bhalla and F. J. Giles, Br. J. Cancer, 2009, 100, 1523-1529.

6. X. Y. Huang, Z. J. Shan, H. L. Zhai, L. N. Li and X. Y. Zhang, J. Chem. Inf. Model., 2011, 51, 1999-2006.

7. M. K. Haider, H.-O. Bertrand and R. E. Hubbard, J Chem Inf Model, 2011, 51, 1092-1105.

8. S. Wolf, M. Amaral, M. Lowinski, F. Vallee, D. Musil, J. Guldenhaupt, M. K. Dreyer, J. Bomke, M. Frech, J. Schlitter and K. Geiwert, J. Chem. Inf. Model., 2019, 59, 5135-5147.

9. J. R. Huth, C. Park, A. M. Petros, A. R. Kunzer, M. D. Wendt, X. Wang, C. L. Lynch, J. C. Mack, K. M. Swift, R. A. Judge, J. Chen, P. L. Richardson, S. Jin, S. K. Tahir, E. D. Matayoshi, S. A. Dorwin, U. S. Ladror, J. M. Severin, K. A. Walter, D. M. Bartley, S. W. Fesik, S. W. Elmore and P. J. Hajduk, Chem Biol Drug Des, 2007, 70, 1-12.

10. P.-P. Kung, B. Huang, G. Zhang, J. Z. Zhou, J. Wang, J. A. Digits, J. Skaptason, S. Yamazaki, D. Neul, M. Zientek, J. Elleraas, P. Mehta, M.-J. Yin, M. J. Hickey, K. S. Gajiwala, C. Rodgers, J. F. Davies and M. R. Gehring, J. Med. Chem., 2010, 53, 499-503.

11. L. Zehnder, M. Bennett, J. Meng, B. Huang, S. Ninkovic, F. Wang, J. Braganza, J. Tatlock, T. Jewell, J. Z. Zhou, B. Burke, J. Wang, K. Maegley, P. P. Mehta, M.-J. Yin, K. S. Gajiwala, M. J. Hickey, S. Yamazaki, E. Smith, P. Kang, A. Sistla, E. Dovalsantos, M. R. Gehring, R. Kania, M. Wythes and P.-P. Kung, J Med Chem, 2011, 54, 3368-3385.

12. P.-P. Kung, P.-J. Sinnema, P. Richardson, M. J. Hickey, K. S. Gajiwala, F. Wang, B. Huang, G. McClellan, J. Wang, K. Maegley, S. Bergqvist, P. P. Mehta and R. Kania, Bioorg Med Chem Lett, 2011, 21, 3557-3562.

13. J. T. Ernst, M. Liu, H. Zuccola, T. Neubert, K. Beaumont, A. Turnbull, A. Kallel, B. Vought and D. Stamos, Bioorg. Med. Chem. Lett., 2014, 24, 204-208.

14. J. T. Ernst, T. Neubert, M. Liu, S. Sperry, H. Zuccola, A. Turnbull, B. Fleck, W. Kargo, L. Woody, P. Chiang, D. Tran, W. Chen, P. Snyder, T. Alcacio, A. Nezami, J. Reynolds, K. Alvi, L. Goulet and D. Stamos, J. Med. Chem, 2014, 57, 3382-3400.

15. D. J. Newman and G. M. Cragg, Journal of Natural Products, 2016, 79, 629-661.

16. B. Haefner, Drug discovery today, 2003, 8, 536-544.

17. D. J. Faulkner, Antonie van Leeuwenhoek, 2000, 77, 135-145.

18. C. Alves, J. Silva, S. Pinteus, H. Gaspar, M. C. Alpoim, L. M. Botana and R. Pedrosa, Frontiers in Pharmacology, 2018, 9.

19. T. F. Molinski, D. S. Dalisay, S. L. Lievens and J. P. Saludes, Nature Reviews Drug Discovery, 2008, 8, 69-85.

20. M. S. Butler, A. A. B. Robertson and M. A. Cooper, Nat. Prod. Rep., 2014, 31, 1612-1661.

21. G. R. Marshall, Ann. Rev. Pharmacol. Toxicol., 1987, 27, 193-213.

22. N. Homeyer, F. Stoll, A. Hillisch and H. Gohlke, J. Chem. Theory Comput., 2014, 10, 33313344.

23. N. T. Mai, N. T. Lan, T. Y. Vu, P. T. Mai Duong, N. T. Tung and H. T. Thu Phung, J. Mol. Graph. Modell., 2020, 100, 107648.

24. S. T. Ngo, N. Quynh Anh Pham, L. Thi Le, D.-H. Pham and V. V. Vu, J. Chem. Inf. Model., $2020,60,5771-5780$. 
25. P.-T. Tran, V.-H. Hoang, J. Lee, T. T. T. Hien, N. T. Tung and S. T. Ngo, RSC Adv, 2019, 9, $29619-29627$.

26. W. Yu and A. D. MacKerell, in Antibiotics: Methods and Protocols, ed. P. Sass, Springer New York, New York, NY, 2017, DOI: 10.1007/978-1-4939-6634-9_5, pp. 85-106.

27. S. T. Ngo, H. M. Hung and M. T. Nguyen, J. Comput. Chem., 2016, 37, 2734-2742.

28. S. T. Ngo, T. H. Nguyen, N. T. Tung, P. C. Nam, K. B. Vu and V. V. Vu, J. Comput. Chem, 2020, 41, 611-618.

29. S. T. Ngo, J. Comput Chem, 2021, 42, 117-123.

30. N. T. Lan, K. B. Vu, M. K. Dao Ngoc, P.-T. Tran, D. M. Hiep, N. T. Tung and S. T. Ngo, J. Mol. Graph. Model., 2019, 93.

31. N. T. Mai, N. T. Lan, T. Y. Vu, P. T. M. Duong, N. T. Tung and H. T. T. Phung, J. Mol. Graph. Model., 2020, 100.

32. C. D. Tuan, T. T. T. Hoa, T. T. T. Van, N. M. Anh, H. T. H. Lien, L. T. H. Minh, P. V. Cuong, D. A. Duy, Y. H. Kim, D. V. Chuc, N. V. Khai, N. V. Hung and D. T. M. Huong, Vietnam J. Mar. Sci. Technol., 2020, 20, 1-10.

33. D. T. Quynh, C. D. Tuan, L. K. Huyen, D. V. Chuc, H. T. H. Lien, L. T. H. Minh, P. V. Cuong, Y. H. Kim, N. V. Hung and D. T. M. Huong, Vietnam J. Sci. Technol., 2020, 58.

34. B. T. N. Quynh, C. D. Tuan, H. T. H. Lien, D. A. Duy, N. M. Anh, V. T. Quyen, P. T. Dao, L. T. H. Minh, D. T. M. Huong, P. V. Cuong, K. T. Quan, N. Q. Tuan, J. W. Chae, H. y. Yun, M. K. Na, Y. H. Kim, D. V. Chuc, N. T. Son and N. V. Hung, presented in part at the 8th International Conference on the Development of Biomedical Engineering (BME8), Ho Chi Minh City, 07/2020, 2020.

35. O. Trott and A. J. Olson, J. Comput. Chem., 2010, 31, 455-461.

36. G. M. Morris, R. Huey, W. Lindstrom, M. F. Sanner, R. K. Belew, D. S. Goodsell and A. J. Olson, J. Comput. Chem., 2009, 30, 2785-2791.

37. G. Jones, P. Willett, R. C. Glen, A. R. Leach and R. Taylor, J. Mol. Biol., 1997, 267, 727-748.

38. S. T. Ngo, N. Hung Minh, H. Le Thi Thuy, Q. Pham Minh, T. Vi Khanh, T. Nguyen Thanh and V. Van, RSC Adv, 2020, 10, 40284-40290.

39. M. Q. Pham, K. B. Vu, T. N. Han Pham, L. T. Thuy Huong, L. H. Tran, N. T. Tung, V. V. Vu, T. H. Nguyen and S. T. Ngo, RSC Adv, 2020, 10, 31991-31996.

40. A. Nurisso, J. Bravo, P.-A. Carrupt and A. Daina, J. Chem. Inf. Model., 2012, 52, 1319-1327.

41. M. J. Abraham, T. Murtola, R. Schulz, S. Páll, J. C. Smith, B. Hess and E. Lindahl, SoftwareX, 2015, 1-2, 19-25.

42. A. E. Aliev, M. Kulke, H. S. Khaneja, V. Chudasama, T. D. Sheppard and R. M. Lanigan, Proteins: Struct., Funct., Bioinf., 2014, 82, 195-215.

43. W. L. Jorgensen, J. Chandrasekhar, J. D. Madura, R. W. Impey and M. L. Klein, J. Chem. Phys., 1983, 79, 926-935.

44. J. Wang, W. Wang, P. A. Kollman and D. A. Case, J Mol Graph Model, 2006, 25, 247-260.

45. D. A. Case, I. Y. Ben-Shalom, S. R. Brozell, D. S. Cerutti, T. E. Cheatham, I. Cruzeiro, V.W.D. , T. A. Darden, R. E. Duke, D. Ghoreishi, M. K. Gilson, H. Gohlke, A. W. Goetz, D. Greene, R. Harris, N. Homeyer, Y. Huang, S. Izadi, A. Kovalenko, T. Kurtzman, T. S. Lee, S. LeGrand, P. Li, C. Lin, J. Liu, T. Luchko, R. Luo, D. J. Mermelstein, K. M. Merz, Y. Miao, G. Monard, C. Nguyen, H. Nguyen, I. Omelyan, A. Onufriev, F. Pan, R. Qi, D. R. Roe, A. Roitberg, C. Sagui, S. Schott-Verdugo, J. Shen, C. L. Simmerling, J. Smith, R. SalomonFerrer, J. Swails, R. C. 
Walker, J. Wang, H. Wei, R. M. Wolf, X. Wu, L. Xiao, Y. D.M. and a. K. P.A., University of California, San Francisco, 2018.

46. J. Wang, R. M. Wolf, J. W. Caldwell, P. A. Kollman and D. A. Case, J. Comput. Chem., 2004, 25, 1157-1174.

47. A. W. Sousa da Silva and W. F. Vranken, BMC Research Notes, 2012, 5, 1-8.

48. H. Zhang, C. Yin, Y. Jiang and D. van der Spoel, J. Chem. Inf. Model., 2018, 58, 1037-1052.

49. H. Zhang, Y. Jiang, Z. Cui and C. Yin, J. Chem. Inf. Model., 2018, 58, 1669-1681.

50. P. Schrödinger LLC, Schrödinger Release 2020-4: Maestro, 2020.

51. The PyMOL Molecular Graphics System, Version 2.4.0, Schrodinger, LLC).

52. L. Zhang, D. Lin, X. Sun, U. Curth, C. Drosten, L. Sauerhering, S. Becker, K. Rox and R. Hilgenfeld, Science, 2020, 368, 409-412.

53. Z. Jin, Y. Zhao, Y. Sun, B. Zhang, H. Wang, Y. Wu, Y. Zhu, C. Zhu, T. Hu, X. Du, Y. Duan, J. Yu, X. Yang, X. Yang, K. Yang, X. Liu, L. W. Guddat, G. Xiao, L. Zhang, H. Yang and Z. Rao, Nat Struct Mol Biol, 2020, 27, 529-532.

54. W. Dai, B. Zhang, H. Su, J. Li, Y. Zhao, X. Xie, Z. Jin, F. Liu, C. Li, Y. Li, F. Bai, H. Wang, X. Cheng, X. Cen, S. Hu, X. Yang, J. Wang, X. Liu, G. Xiao, H. Jiang, Z. Rao, L.-K. Zhang, Y. Xu, H. Yang and H. Liu, Science, 2020, 368, 1331-1335.

55. N. T. Dan, H. D. Quang, V. Van Truong, D. Huu Nghi, N. M. Cuong, T. D. Cuong, T. Q. Toan, L. G. Bach, N. H. T. Anh, N. T. Mai, N. T. Lan, L. Van Chinh and P. M. Quan, Sci Rep, 2020, 10, 11429.

56. N. M. Tam, P. C. Nam, D. T. Quang, N. T. Tung, V. V. Vu and S. T. Ngo, RSC Adv, 2021, 11, 2926-2934.

57. S. T. Ngo, B. K. Mai, P. Derreumaux and V. V. Vu, RSC Adv, 2019, 9, 12455-12461.

58. S. T. Ngo, K. B. Vu, L. M. Bui and V. V. Vu, ACS Omega, 2019, 4, 3887-3893.

59. N. M. Tam, M. T. Nguyen and S. T. Ngo, J. Mol. Graph. Model., 2017, 77, 137-142. 